\title{
Development of Striatal Tissue Implanted Into the Anterior Eye Chamber of Rats
}

\author{
M. Kálmán, A. Tuba, E. Adeghate and Z. Fülöp* \\ 1st Department of Anatomy, Semmelweis University of Medicine, Budapest, Hungary \\ *Present address: Rutgers University, Institute of Animal Behavior, Newark, NJ, USA
}

Transplantation of catecholaminergic tissues into the striatum is a new form of surgical therapy in Parkinson's disease. The superior cervical ganglion is one of the potential graft tissues. After successful transplantation, the grafted ganglion innervates the host tissue. Iridic tissue receives its sympathetic innervation from the superior cervical ganglion and its highly vascularized tissue is a suitable medium for brain transplants. Thus, the striatum transplanted into the anterior eye chamber can provide an "inverse" model for studying the connection that may develop between the sympathetic fibers and the striatal tissue.

Rat embryos (E15 or E16) were removed from the mother under deep ketamine anaesthesia. The ganglion hill (striatal anlage) was dissected out of the brain and implanted by a syringe into the anterior eye chamber of adult rats of either sex which had received ketamine anesthesia following atropine pre-medication. After 2-to-4-week survival periods the host rats were perfused under deep ether narcosis with $4 \%$ buffered paraformaldehyde solution. In about $50 \%$ of cases, we found transplants attached to the iris. After embedding into epoxy resin (Durcupan, Fluka), semithin sections were prepared and stained with toluidine blue or processed for immunohistochemistry.

In the sections stained with toluidine blue, some nucleus-like groups of neurons were seen separated by neuropil and looser networks of neurons. The neurons observed were usually rather large. Their nuclei were especially large as compared to the cytoplasm. The nucleoli were dislocated to the nuclear envelope. The Nissl-substance was dense and well-developed. In our opinion, these alterations must be due to a forced development of neuronal processes accompanied by an enhanced protein synthesis in an environment not optimal for the outgrowth of processes. Astrocytes and satellite glial cells were frequently observed. Myelinated fibers occurred only sporadically. At some points of the transplant's periphery, invasion of macrophages could be seen.

To study the glia and the catecholaminergic structures, semithin sections were processed for postembedding GFAP (glial fibrillary acidic protein) and $\mathrm{TH}$ (tyrosine hydroxylase) immunohistochemistry, respectively. Before the immunoreaction, the sections were etched by sodium ethanolate to eliminate the epoxy resin. Incubations were performed in a humid chamber at room temperature. Non-specific reactions were diminished by normal goat serum. Monoclonal mouse anti-GFAF (Boehringer, Mannheim) and polyclonal rabbit anti-TH (EugeneTech) were applied as primary antibodies (for $2 \mathrm{~h}$, in dilutions 1:100 and 1:500, respectively). The immunoreaction was developed by biotinylated secondary antibodies and avidin-horseradish peroxidase complex (each for $20 \mathrm{~min}$, diluted at 1:100), and was then visualized by diaminobenzidine reaction. For comparison, some slices of substantia nigra and cerebellum were also incubated with anti-TH and antiGFAP, respectively. Parallel sections of transplants were processed without primary antibodies. No diaminobenzidine reaction product was found in these cases.

A distinct GFAP-immunoreaction was found throughout the transplants. Characteristic connections between astroglial processes and blood vessels were frequently seen. No peripheral glial barrier developed against the surrounding non- 
neural host tissue.

In only a few sections could we observe small diaminobenzidine-positive dots after $\mathrm{TH}$ immunostaining. Whether they really represent nerve fibers or endings, remains to be clarified by forthcoming electron microscopic investigations, although sometimes the localization of the reaction product (in the wall of blood vessels) was characteristic for noradrenergic nerve fibers.
No intrinsic neuron-like TH-immunopositive structures were observed in the transplants, so an extrinsic, i.e. superior cervical ganglion, origin of $\mathrm{TH}$-immunopositive elements can be supposed. In conclusion, the catecholaminergic innervation of striatal explants appears to be occasional and sporadic rather than massive and regular. 

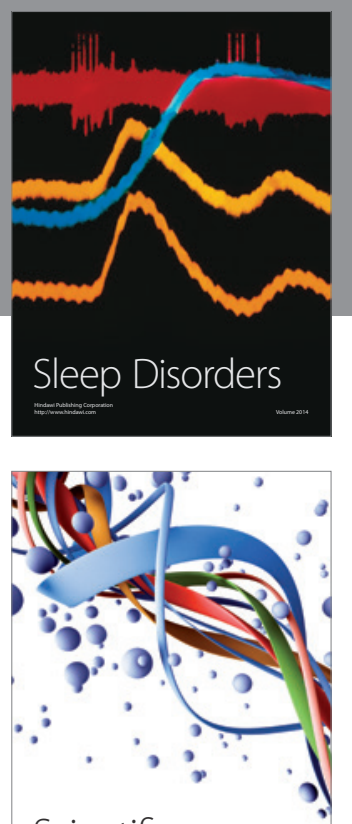

Scientifica
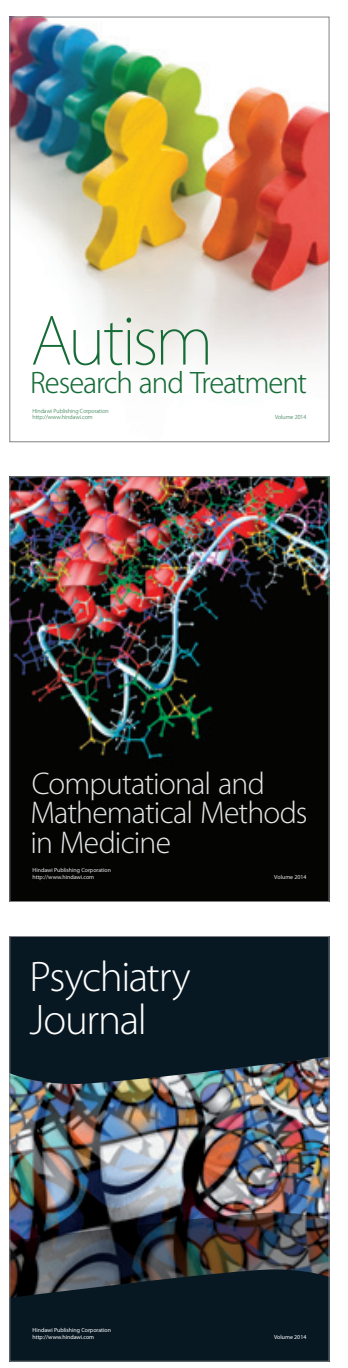
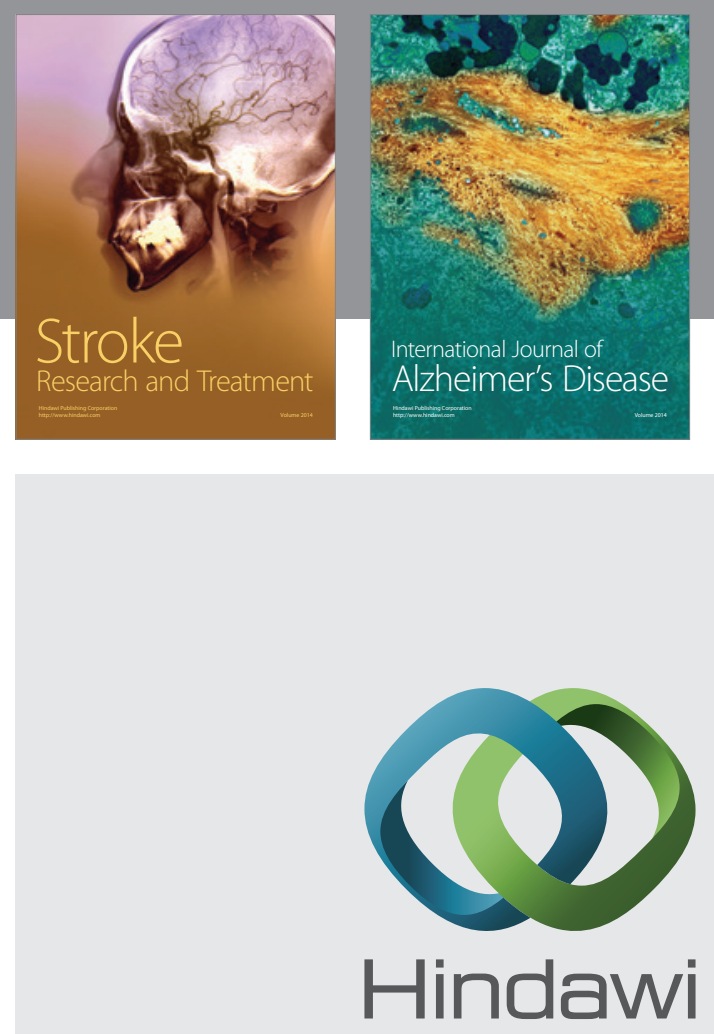

Submit your manuscripts at

http://www.hindawi.com
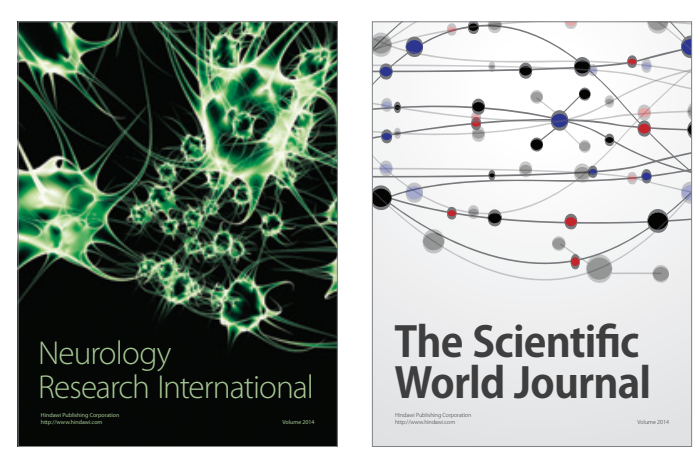

The Scientific World Journal

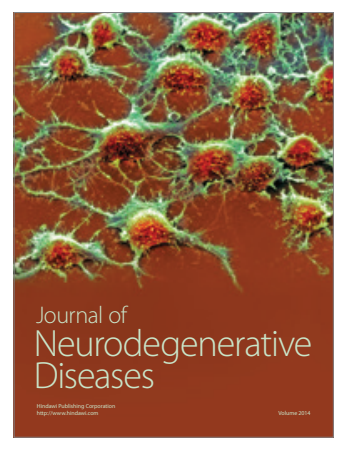

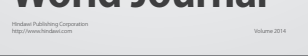

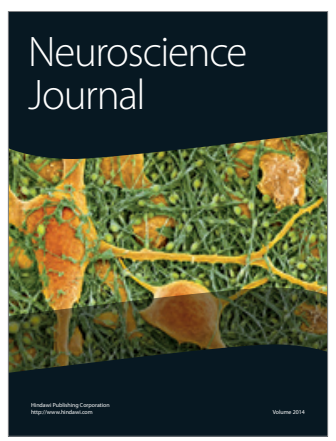

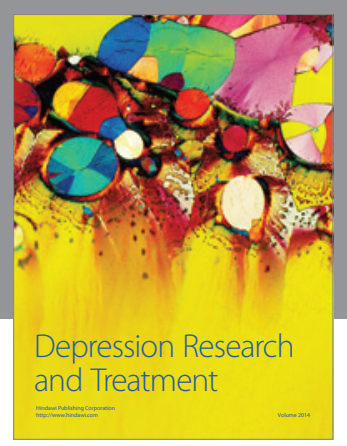
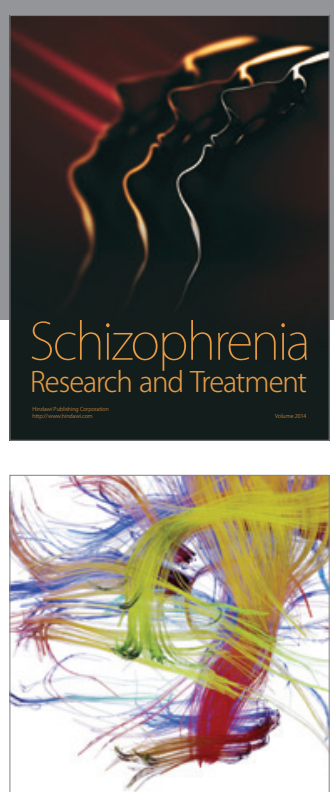

Brain Science

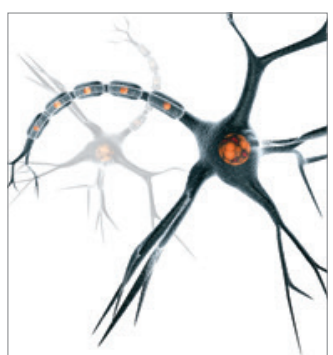

Neural Plasticity
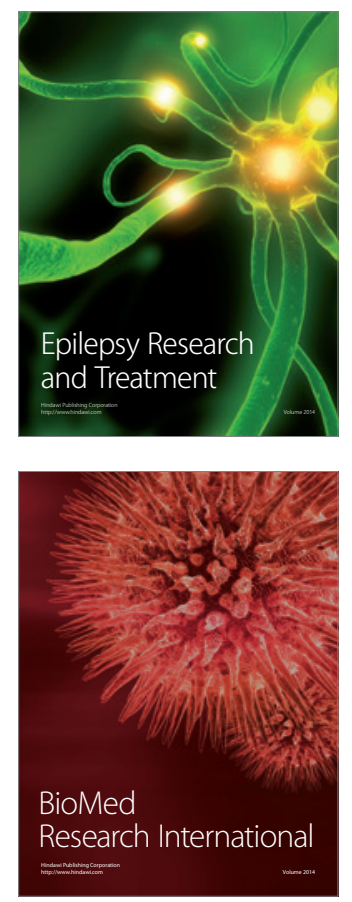

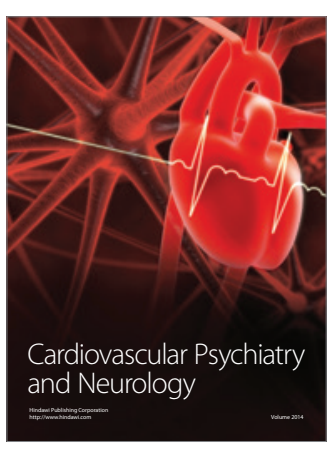

Parkinson's

Disease
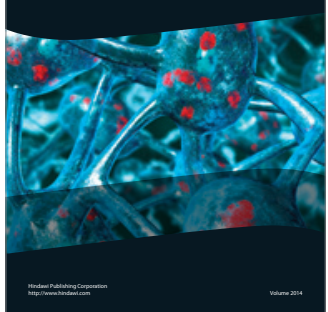